\title{
Effect of Emitter Layer on the Signal Generation of the Active Pixel Sensor
}

\author{
Sangsik Park and Hyungsoo Uh
}

\begin{abstract}
The image sensor in the optical mouse reads the scene of the bottom, and compares it with the last sceneto acquire the moving data. The scene should be read with high frame rate to make the moving of the cursor soft and exact. The high frame rate means the short time to integrate the signal charge. The weak signal causes malfunctioning of the moving of the cursor. The signal should be amplified to ensure the signal to noise ratio. Some type of the pixel in the optical mouse uses a bipolar transistor to amplify the signal.

The conventional bipolar transistor used in the optical mouse has full covering emitter layer on the base layer. The emitter layer helps the bipolar action, but it decreases the signal electron-hole pair generation since the generation occurs in the collector-base junction. We propose the emitter structure which covers only the boundary of the base layer. The photo current of the new structure increased $20 \%$ compared to the conventional structure.
\end{abstract}

Index Terms-Bipolar transistor, emitter, image sensor, photo generation, sensitivity.

\section{INTRODUCTION}

We use mouse to make the cursor on the screen to move softly and exactly. The mouse adopts optical system instead of ball system since the optical system shows even more exact and smooth movement of the cursor on the screen. Every optical system has the function of LED illumination, focusing by lens and image sensing using a sensor. The optical mouse senses scene of the bottom surface about 3,400 6,000 times a second, and produce pixel movements with external oscillator by comparing present image and previous image.

The LED (Light Emitting Diode) is used to illuminate work surface to acquire a high quality image. The power dissipation of LED should be low since wireless mouse is used universally. The power dissipation of LED depends on current of LED, so current of LED should be as possible as low. But the acquired image can be bad under low illumination by a low current LED. Therefore, the sensitivity of the image sensor should be high to acquire a clear image under low illumination.

The frame rate of the capture of the scene of work surface

Manuscript received May 18, 2013; revised July 19, 2013. This work was supported in part by the Korea Department of trade, industry and energy System IC 2015.

Sangsik Park was with Image sensor development group of Samsung Electronics. He is now a professor of department of Electronics Engineering of Sejong University, Kunja-dong, Seoul, 143-747 Korea (email: sspark@sejong.ac.kr).

Hyungsoo Uh was with semiconductor process development group of Samsung Electronics. He is now a professor of department of Electronics Engineering of Sejong University, Kunja-dong, Seoul, 143-747 Korea (email: hsuh@sejong.ac.kr).. should be high to get a smooth and exact movement of the cursor. That means the charge integration time of the photo detector is short compared to the visible image sensor. The photodiode of general image sensor used for the mobile phone integrates signal charge for above 10ms 30ms.[1] But the signal integration time of photo detector of mouse image sensor is only a few hundred micro second. [2] The integrated signal charge in a period is very small compared to the general image sensor, so the $\mathrm{S} / \mathrm{N}$ ratio (signal to noise ratio) can be too low to get exact moving vector.[3] The sufficient signal to get clear moving vector can be acquired by amplification of signal charge generated in the photo detector of the image sensor. The emitter current is amplified $\beta$ times of the base current in a bipolar transistor. The signal appearing in the emitter terminal will be $\beta$ times of the signal generated in the base - collector pn junction.

The problem caused by the low illumination and the short integration time will be resolved at the same time by the amplification. The higher current gain of bipolar transistor realizes and higher frame rate under the illumination of lower LED current.

Fig. 1 shows the simplified structure of a pixel used in the image sensor of the optical mouse. The photo detector is the collector-base junction of a pnp bipolar transistor and current source means the photo current generated in the base-collector pn junction. The electron-hole pairs are generated in the base - collector pn junction that means current flows from n-type base to p-type collector by the incident light on the base - collector pn junction. The electron goes to the n-type base, and the hole moves to the p-type collector. These flows form the base current, so $\beta$ times of the base current flows in the emitter terminal. The amplified emitter current is integrated in a capacitor located between emitter and power supply terminal. The voltage of the capacitor becomes higher as light incident on this pixel increases during the period by the emitter current.

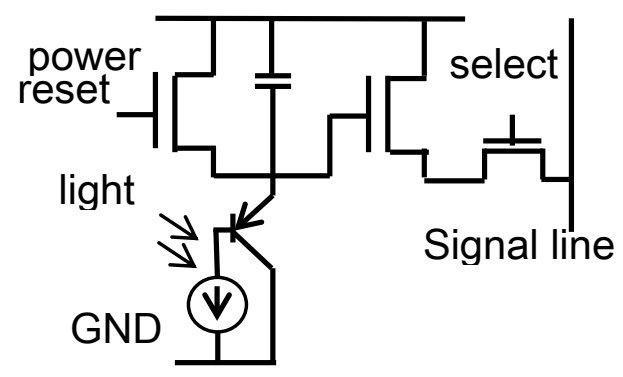

Fig. 1. Simplified structure of a pixel used in the image sensor of the optical mouse. The current source means the photo current generated in the basecollector pn junction. Amplified emitter current is integrated in the capacitor. 
The operation sequence of this pixel is as following.

1) A reset pulse is applied to the reset terminal to make the voltage of the capacitor be zero.

2) This pixel waits one frame to integrate the signal charge in the capacitor. Other pixels are read in this time.

3) After one period, this pixel is read. The "high" voltage is applied to select transistor to connect this pixel to the common vertical signal line. The signal voltage appeared in signal line is sampled and stored.

4) A reset pulse is applied to the reset terminal to make the voltage of the capacitor be zero for the next field. This is the same sequence in the step 1.

In chapter II, the experiment to get high signal current is presented. The results are analyzed in point of the photosensitive depth. The characteristics of the mouse adopting this photo detector are shown in chapter III. Finally, the conclusions are presented in chapter IV.

\section{EXPERIMENTS AND DISCUSSION}

The performance of each photo detector were tested using 18 pixel $\times 18$ pixel image sensor for optical mouse which is composed of pixels, timing generation circuits, A/D converters and data processing blocks. The image sensor was manufactured using $0.18 \mu \mathrm{m}$ design rule standard CMOS process. The concentration of the n-well is $5 \times 10^{16} / \mathrm{cm}^{3}$ on surface and $3 \times 10^{15} / \mathrm{cm}^{3}$ in $0.7 \mu \mathrm{m}$ depth from the silicon surface. The concentration becomes lower as the depth be deeper [4].

The layouts of the pixel are shown in Fig. 2. The conventional pixel is shown in (a), and proposed pixel is shown in (b). The inner contacts are emitter contacts, outer contacts are base contacts. Base terminal has just 1 contact for the purpose of only test, since there is no current flowing in the base terminal. The Amplified current flows form emitter to collector by the photo-generated base current. The pitch of the pixel is $52 \mu \mathrm{m} \times 52 \mu \mathrm{m}$, and the aperture ratio is $64.5 \%$. The area of photo detector is designed much more wide compared to that of image sensors used in the mobile phone since charge integration time is very short compared to that.

There is no whole covered emitter layer in the surface of the proposed layout. The photo generating area can be widened since the emitter region disappeared.

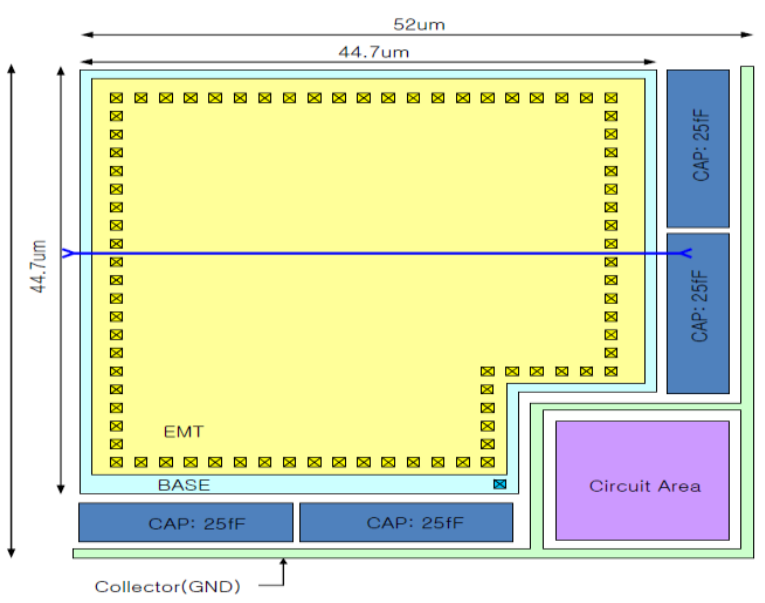

(a)

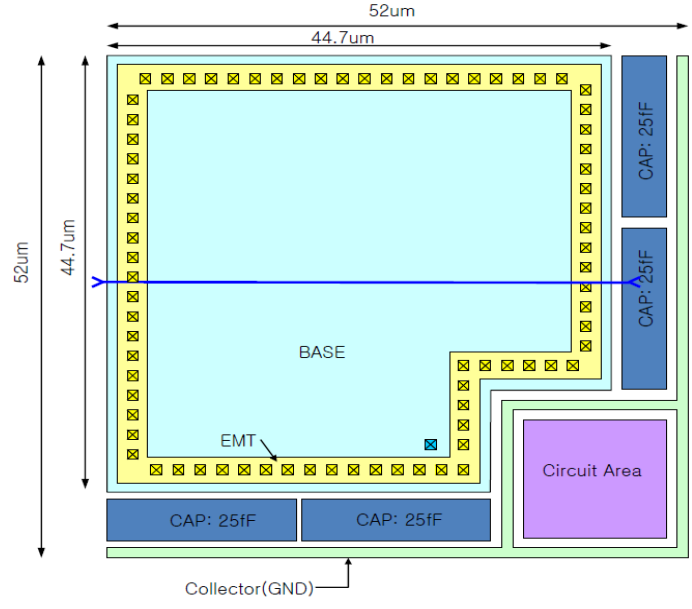

(b)

Fig. 2. The layout of a pixel. (a) conventional structure (b) proposed structure. The inner contacts are emitter contacts, outer contacts are collector contacts. The capacitors are for the signal collection.

Fig. 3 shows the cross section view of the photo transistors in pixels used in this experiment. The light is incident on pn junction composed of base and collector. Type A (a) has the emitter layer on the entire surface of the photo transistor, on the other hand Type B (b) has no emitter layer on entire surface. Type B has just guard-ring type emitter for the operation of the bipolar transistor.

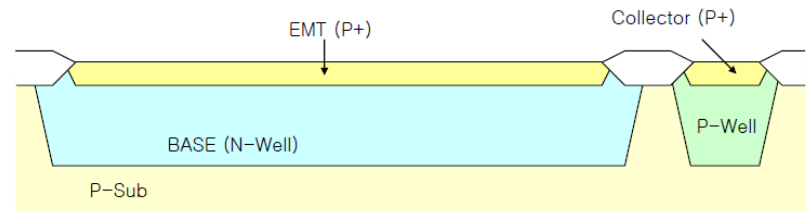

(a)

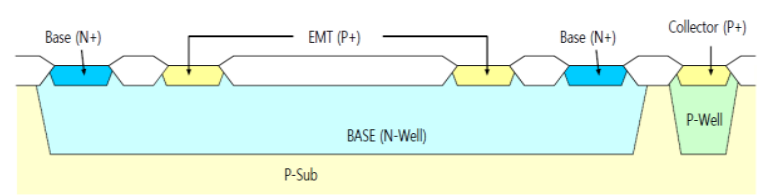

(b)

Fig. 3. The vertical cross section view of photo transistors in pixels used in this experiment. (a) conventional structure has whole covered emitter on the surface, (b) proposed photo-transistor has no emitter layer on entire surface but has guard ring type emitter on boundary of the base.

The measured photo currents of conventional structure in Fig 3 is $3.12 \mathrm{nA}$, and that of the proposed structure is 3.72 nA. The photo currents were measured under LED illumination of 60 lux and the wavelength of incident light was $639 \mathrm{~nm}$. [5]

The photons absorbed in the whole covered emitter layer of type A generates photo induced electron and hole pairs in the emitter layer. [5] But the generated electrons in the emitter $\mathrm{p}+$ layer are recombined by the electrons since the emitter layer is very high doped p-type, that means there are very concentrations of holes in the layer. So the electrons generated in the emitter layer can not go to base region, can not contribute to a signal charge, but disappears. The electrons can not form base current. The type B structure has no emitter layer on entire surface but has guard ring type emitter on boundary of the base. There is no loss of photo generated electrons in the type B structure. That's the reason why the photo current of the type B is higher than that of type A. 


\section{Performance of IMAge Sensor}

The sequence of the image signal flow of the mouse using this structure is shown in Fig. 4. The LED illuminates the bottom surface, and the bottom scene is focused on the image sensor by the optical lens. Then the image sensor which has 18 pixel $\times 18$ pixel captures the image of the bottom as the photo currents integrated in capacitors at a rate of 3,400 6,000 times a second. The frame rate depends on the speed of the mouse. The base current is amplified to the emitter current and is converted to analog voltages in capacitors, and analog voltages are converted to digital value by the built in Analog-digital converter.

Then the digital image data is processed to find out the optimized moving vector by comparing present image and previous image. This moving vector is transferred to the PC to make the cursor.

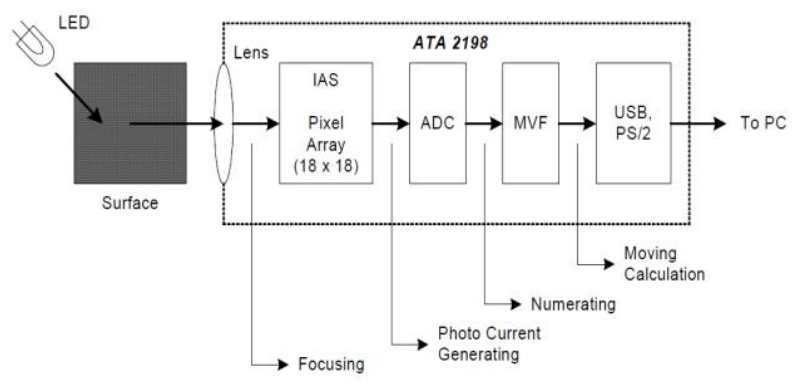

Fig. 4. The sequence of the image signal flow of the mouse using this structure.

The PCB board of the mouse using this photo transistor as a photo detector is shown in Fig. 5. This image sensor calculates maximum \pm 3 pixel movement per frame, and produce maximum 10,200 pixel movements a second with external $12 \mathrm{MHz}$ oscillator. The speed of mouse motion is up to $25 \mathrm{inch} / \mathrm{sec}$ at this oscillator frequency. The sampling rate of motion detector is $50 \mathrm{kHz}$ and the space resolution is 1600 cpi (counter per inch).

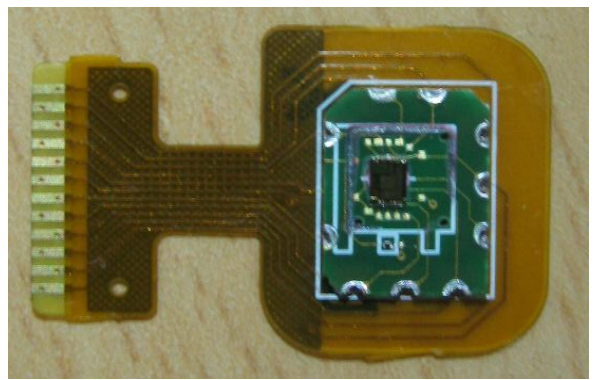

Fig. 5. The PCB board of the mouse using this photo transistor as a photo detector.

\section{CONCLUSION}

A new structure of photo transistor was proposed to get higher sensitivity for image sensor used in the optical mouse. The proposed photo-transistor has no emitter layer on entire surface but has guard ring type emitter on boundary of the base. The performance of proposed photo detector was verified using 18 pixel $\times 18$ pixel image sensor in the optical mouse which is composed of pixels, timing generation circuits, A/D converters and data processing circuits. The image sensor was manufactured using $0.18 \mu \mathrm{m}$ design rule standard CMOS process. The sensitivity was increased $20 \%$ in structure which has no emitter layer on entire surface. There is no loss of photo generated electrons in the proposed structure. That is the reason why the photo current of the proposed structure is higher than that of conventional one. This image sensor was mounted in the optical mouse. The high sensitivity allowed the speed of mouse motion up to $25 \mathrm{inch} / \mathrm{sec}$.

\section{ACKNOWLEDGMENT}

This work was supported by the System IC 2015 of Korea Department of trade, industry and energy.

\section{REFERENCES}

[1] P. Donegan, E. Fox, B. Li, M. Sonder, F. Feng, M. Kiik, and S. Xie, "A 4M pixel Image Sensor fot High Speed Image Capture," Advanced Image Sensor Workshop, pp. 185-188, 2005.

[2] A. Krimski, E. Bock, N. Tu, D. Blerkom, and E. Fossum, "A High Speed 240-Frame/s, 4.1-Mpixel CMOS Sensor," IEEE Transactions on ED, vol. 50, no. 1, pp. 130-135, 2003.

[3] H. Tian, B. Fowler, and A. Gamal, "Analysis of Temporal Noise in CMOS photodiode Active Pixel Sensor," IEEE Journal of Solid-state Circuits, vol. 36, no. 1, pp. 92-101, 2001.

[4] H. Chien, S. Wuu, D. Yaung, C. Cheng, J. Lin, C. Wang, C. Chang, and Y. Hsiao, "Active Pixel Image sensor scale down in $0.18 \mu \mathrm{m}$ CMOS Technology," 2002 IEDM, pp. 813-816, 2002.

[5] L. J. Kozlowski, J. Luo, and A. Tomasini, "Performance Limits in visible and infrared image sensors," IEDM Tech. Digest, pp. 867-870, Dec. 1999.

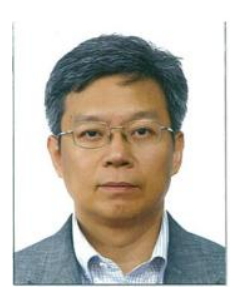

Sangsik Park was born in Korea at 1961. He was with Image sensor development group of Samsung Electronics. He is now a professor of department of Electronics Engineering of Sejong University. His major field is image sensor. 